\title{
Retention Management im Krankenhaus - Arbeiten im Spannungsfeld der Gesundheitsversorgung
}

\author{
Christian Kempny ${ }^{1}$ Christian Breimann² \\ Online publiziert: 30. Juni 2020 \\ (c) Der/die Autor(en) 2020
}

\section{Zusammenfassung}

Dieser Beitrag der Zeitschrift „Gruppe. Interaktion. Organisation. (GIO)“ beschreibt Herausforderungen für das Retention Management in Krankenhäusern und belegt diese mit Daten aus einer empirischen Studie. Die Arbeit in einem Krankenhaus beschreibt einen besonderen Arbeitskosmos, in dem nicht nur die Motive der Mitarbeiter, sondern auch die besondere Stellung des Patienten, die Organisationsform „Krankenhaus“ wesentlich von anderen Organisationen im wirtschaftlichen Kontext abgrenzt. Dennoch müssen sich auch Krankenhäuser dem Fachkräftemangel und der demografischen Entwicklung in Deutschland stellen. Um ein funktionierendes Retention Management in ein Krankenhaus zu integrieren und den Problemen vorzubeugen, müssen zunächst die drei Spannungsfelder „Wirtschaftlichkeit“, „Interkulturalität“ und „Stress“ betrachtet werden. Diese stellen Mitarbeiter in einem Krankenhaus immer wieder, im ohnehin arbeitsintensiven Alltag, vor große Herausforderungen. Es konnten verschiedene Handlungskonzepte entwickelt werden, welche es der Organisation „Krankenhaus“ ermöglicht, gegen die Spannungsfelder zu arbeiten und Mitarbeiter langfristig an das eigene Unternehmen zu binden.

Schlüsselwörter Arbeitsbelastung $\cdot$ Interkulturalität $\cdot$ Retention Management $\cdot$ Spannungsfelder $\cdot$ Sprache $\cdot$ Stress . Wirtschaftlichkeit

\section{Retention management in the hospital setting-working in a field of tension}

\begin{abstract}
This contribution from the journal „Gruppe. Interaktion. Organisation. (GIO)“ describes challenges for retention management in hospitals and backs them up with data from an empirical study. The workplace in a hospital describes a special working environment in which not only the motives of the employees, but also the special position of the patient, the organizational form "hospital“ is essentially differentiated from other organizations in the economic context. Nevertheless, hospitals must also face the lack of skilled employees and the demographic development in Germany. In order to integrate a functioning retention management into a hospital and to prevent these problems, the three issues "economic efficiency", "interculturality" and "stress" must be considered first. These issues pose a constant challenge to employees in a hospital, in their already labor-intensive everyday life. Different concepts of action were developed, which allows the organization "hospital" to work against the three issues and to bind employees in the long term to their own company.
\end{abstract}

Keywords Efficiency $\cdot$ Fields of tension $\cdot$ Interculturality $\cdot$ Language $\cdot$ Retention Management $\cdot$ Stress $\cdot$ Workload

Christian Kempny

Christian.Kempny@hshl.de

Christian Breimann

christian.breimann@gmail.com
1 Hochschule Hamm-Lippstadt, Marker Allee 76-78, 59063 Hamm, Deutschland

2 Friedrich-Hebbel-Straße 1, 46537 Dinslaken, Deutschland 


\section{Einleitung}

Die Tätigkeit in einem Krankenhaus ist eine besondere Arbeit, die nicht durch gute Verdienstmöglichkeiten oder durch hohes soziales Ansehen bestimmt ist, sondern es handelt sich bei der Tätigkeit als Angestellter im medizinischen Bereich gewissermaßen um eine Berufung, sich um Menschen in Ausnahmesituationen zu kümmern und sich diesen anzunehmen. Jedoch ist es nicht möglich in einer Zeit, in der Kostendruck und Wettbewerbsorientierung in vielen Bereichen Einzug halten, sich nur auf eine ideologische Hilfsbereitschaft zu beziehen. Vielmehr befinden sich Mitarbeiter in einem Krankenhaus immer wieder auf einer Gratwanderung zwischen Patientenwohl und Wirtschaftlichkeit (Behar et al. 2016). Natürlich sorgen medizinethische Grundregeln dafür, dass sich das Personal im Zweifel immer für den Patienten und gegen wirtschaftliche Interessen entscheiden sollte, dennoch bringt dieser Spannungszustand neue Herausforderungen für Mitarbeiter in einem Krankenhaus (Staar und Kempny 2019; Staar et al. 2018). Viele weitere Hindernisse, wie kulturelle und sprachliche Barrieren, kommen zudem immer stärker auf das medizinische Personal zu, welche das Spannungsfeld im Krankenhaus immer mehr bestimmen. Somit ergeben sich in einem Krankenhaus besonders drei Spannungsfelder, welche Teil dieses Artikels werden sollen. Die Notwendigkeit sich wirtschaftlicher ausrichten zu müssen, ist hierbei das erste Spannungsfeld. Das zweite Spannungsfeld ist die Interkulturalität in Bezug auf das Personal, aber auch in Bezug auf die Patienten. Das dritte Spannungsfeld betrachtet gesondert die Mediziner in einem Krankenhaus und geht auf das Stresserleben dieser ein.

Um sich gegenüber den Spannungsfeldern adäquat zu positionieren, müssen Krankenhäuser sich in eine Wandelprozess begeben, um die Spannungsfelder zu minimieren oder auszugleichen (Staar et al. 2018). Es ist somit wichtig, neue Sichtweisen und Ausrichtungen der Organisation „Krankenhaus“ vorzunehmen. So müssen neue Handlungskonzepte für die neuen Herausforderungen in Bezug auf das Personal, die wirtschaftliche Struktur und auch für die Patientenbetreuung gefunden werden. Seit der Umstellung der Vergütungspauschalen für Krankenhäuser müssen personalpolitische und wirtschaftliche Entscheidungen immer mehr in den Vordergrund des Krankenhausmanagements rücken. Dies beeinflusst zum einen die Behandlung der Patienten, aber auch das Leitbild und das Selbstverständnis der Mitarbeiter in einem Krankenhaus. Es besteht beispielsweise nicht mehr die Möglichkeit, unabhängig von entstehenden Kosten, die Patienten zu behandeln (Behar et al. 2016). Dass diese Entwicklung derzeit nicht in allen Krankenhäusern funktioniert oder ein Wandel nur in Grundzügen begonnen hat, lässt sich an den Krankenhäusern zeigen, die nicht in eine gewinnbringende oder kostenneutrale Zone kommen (Blum et al. 2013). Der Bedarf für unternehmerisches Verhalten in Krankenhäusern besteht und ist für die Organisation, die Mitarbeiter und letzten Endes auch für die Patienten essenziell, um ein Fortbestehen einzelner Häuser zu gewährleisten. Ein Wandel der Unternehmenskultur, der Personalpolitik und der wirtschaftlichen Ausrichtung des Unternehmens Krankenhaus, muss daher stattfinden und sollte sich hierbei besonders auf „Corporate Entrepreneurship“ und „Retention Management“ im Bereich Krankenhaus und im Gesundheitssektor fokussieren.

Dieser Artikel soll dem Leser einen Überblick über die Besonderheiten des Unternehmenskontextes Krankenhaus und die verschiedenen Spannungsfelder geben. Um die Erkenntnisse möglichst praxisnah zu gestalten, wurden in diesen Artikel zwei Studien eingebunden. Das erste Spannungsfeld „Wirtschaftlichkeit“ wird durch eine Interviewerhebung und das dritte Spannungsfeld Stress bei Medizinern durch eine quantitative Fragebogenerhebung gestützt. Im Folgenden sollen die einzelnen Studien und die theoretische Erarbeitung des zweiten Spannungsfeldes „Interkulturalität" einzeln erläutert werden. Darauf folgt die Einführung des Konzeptes „Retention Management“ und Praxisimplikationen, welche aus den drei Spannungsfeldern und den Erhebungen resultieren.

\section{Besonderheit von medizinischen Berufen}

Bevor die verschiedenen Spannungsfelder im Kontext der Organisation Krankenhaus betrachtet werden können, muss zunächst die Besonderheit von medizinischen Berufen gegenüber anderen Berufsgruppen herausgestellt werden. Bei dem System Krankenhaus handelt es sich um ein sehr bürokratisches und strikt organisiertes System (Staar et al. 2014). Diese starren und traditionsreichen Organisationsstrukturen, welche unter anderem in den klaren, hierarchischen Linien im Personalgeflecht erkennbar werden, begegnen auch immer wieder in Form von klaren Regeln und Verfahrensschritten den Patienten und Angehörigen im Krankenhaus. Dort können sie bei Patienten Unsicherheit und Unverständnis auslösen (Staar und Kempny 2019). Seit der Umstellung der Fallabrechnung in den Krankenhäusern kommt es zwangsläufig zu Change-Prozessen, die nicht von allen Mitarbeitern und allen Patienten gleichsam positiv aufgenommen werden. So kann es zu Konflikten und Unzufriedenheit aufgrund dieser Prozesse kommen (Albrecht und Töpfer 2017). Einer dieser veränderten Prozesse ist beispielsweise die Dokumentationspflicht bei Medizinern und Mitarbeitern in der Pflege. Die Dokumentationspflicht ist schon lange ein Instrument, welches bei der medizinischen Behandlung genutzt wird (vgl. Bayer 2018). Durch das vorherrschende Motto „Was nicht aufgeschrieben worden ist, ist nicht gemacht worden“, sorgen Mediziner und das Pfle- 
gepersonal nicht nur für eine qualitativ hochwertige Behandlung, sondern sichern sich auch rechtlich bei Behandlungen ab. Eine Ausweitung dieser Dokumentation kann bei einigen Medizinern und bei dem Pflegepersonal auf Hindernisse und Widerspruch treffen. Aufgrund der ohnehin eng bemessenen Zeit von Medizinern und des Pflegepersonals, erscheint eine umfangreiche und genaue Dokumentation als eine weitere Belastung. Vor dem Kontext des neuen Vergütungssystems bedeutet eine umfangreiche und genaue Dokumentation der Behandlungsschritte und der Aufzeichnung von Gesundheitsdaten der Patienten eine höhere bzw. angemessene Vergütung (Leiner 2012). Mit ausführlichen Dokumentationen können Abrechnungen unter Umständen wesentlich höher ausfallen, da besondere Behandlungen zusätzlich abgerechnet werden können oder Gesundheitsdaten des Patienten auf eine besonders intensive Pflege hinweisen (Staar et al. 2018). Die Dokumentationspflicht zeigt beispielhaft auf, wie neue Prozesse und verbesserte Methoden in der medizinischen Behandlung zwar für die Organisation einen finanziellen Mehrwert bedeuten können, aber gleichzeitig bei der Belegschaft auf Gegenwehr stoßen.

Betrachtet man verschiedene Bereiche in der Organisation Krankenhaus, trifft man nicht nur auf unterschiedliche Ausbildungsarten, wie zum Beispiel ein langes akademisches Studium oder eine sehr praktische Ausbildung, sondern auch auf verschiedene motivationale Hintergründe, wie beispielsweise Ansehen, Altruismus, biologisches und anatomisches Interesse und finanzielle Anreize, die die Arbeit der Mitarbeiter im Krankenhaus begleiten (Staar et al. 2018).

Betrachtet man die Gruppe der Pflegekräfte in Bezug auf die Wahl des Berufes, können hier nicht ernsthaft die finanziellen Anreize oder vermeintlich gute Arbeitszeiten als Begründung aufgeführt werden. Vielmehr handeln Pflegekräfte stärker aus einem altruistisch-helfenden Motiv heraus, als es in anderen Berufsgruppen zu finden ist (Staar et al. 2018). Bei relativ geringer Entlohnung, einem extrem hohen Arbeitspensum und hoher körperlicher Beanspruchung, leisten diese Mitarbeiter in einem Krankenhaus hoch qualifizierte Arbeit.

Eine weitere Gruppe, die für diese Arbeit betrachtet werden soll, sind die Ärzte. Zwischen diesen gibt es eine klare Unterscheidung in der hierarchischen Struktur. Während die Assistenzärzte bei einem vergleichsweise geringen Gehalt und hoher Arbeitsbelastung recht wenig Entscheidungsfreiraum besitzen, haben Chefärzte und Oberärzte einen anderen finanziellen Anreiz und größere Freiräume bei der Arbeit. Zwar lassen sich auch bei diesen Personengruppen durchaus besondere Motive finden, die sich auf die helfende und unterstützende Arbeit mit Menschen beziehen, jedoch können auch hier die Aufstiegs- und Verdienstmöglichkeiten als Motive für die Berufswahl gesehen werden (Staar et al. 2018).
Der Fachkräftemangel stellt Krankenhäuser vor immer größere Herausforderungen (Schmidt et al. 2012). Eine hohe Fluktuation und der Wegfall von qualifiziertem Personal durch den demografischen Wandel in Deutschland führt zu Diskontinuitäten in der Versorgung, Wissensverlust über Prozesse und Abläufe im Krankenhaus und dadurch auch zu Störungen des Betriebsklimas und der Abläufe (Ulatowski 2013). Die hohe Fluktuation und der altersbedingte Wegfall von Personal können derzeit nicht durch neue Auszubildende, Studierende oder Berufsanfänger gedeckt werden (Schmidt et al. 2012). Ein ähnliches Problem zeigt sich auch bei niedergelassenen Ärzten in ländlichen Bereichen. Auch die Zuwanderung von qualifiziertem Personal ist hier keine ausreichende Option, um den Mangel in der Personalstruktur zu decken (Ulatowski 2013). Zudem kommen bei diesen zugewanderten Experten weiter Schwierigkeiten bei der Beschäftigung im Kontext der Gesundheitsversorgung hinzu (Staar und Kempny 2019). Um diesem Wandel entgegenzuwirken, müssen Krankenhäuser aktiv qualifiziertes Personal an das eigene Haus binden und zudem weiteres Personal rekrutieren, um den Fachkräftemangel decken zu können (Haupt und Bouncken 2014).

\section{Spannungsfeld Krankenhaus - Betriebswirtschaft und Medizin}

Neben dem Kampf um qualifiziertes Personal, ist auch die Finanzierung und die wirtschaftliche Ausrichtung des Krankenhauses eine Aufgabe, der sich das Management stellen muss, um langfristig am Markt agieren zu können.

Durch die Kostenreform im Zeitraum zwischen 2003 und 2014 wurde das DRG-Vergütungssystem eingeführt. Bedingt durch die Reform wandelten sich Krankenhäuser in dieser Zeit gezwungenermaßen von Cost-Centern zu Profit-Centern. Die wirtschaftliche Ausrichtung der Häuser gewann nach der Reform essenziell an Bedeutung. Während Wirtschaftlichkeit vor der Reform bedeutete, dass alle Betten in einem Krankenhaus besetzt waren und genügend Patienten im Krankenhaus behandelt wurden, bedeutet Wirtschaftlichkeit im Krankenhauskontext heute, Patienten möglichst effizient zu behandeln, um diese dann auch möglichst schnell entlassen zu können (Staar et al. 2018). Dies führt zu einer Ambulantisierung bei den Operationen, welche diese Behandlungsform zulassen. Außerdem herrscht ein hoher Zeit- und Kostendruck für die Krankenhäuser, ein stärkerer Wettbewerb unter den Häusern und die Notwendigkeit zur laufenden Verbesserung von Prozessen und Abläufen. Jedoch haben viele der Häuser Probleme, den Kostenausgleich oder bestenfalls die Gewinnzone zu erreichen. Nach Zahlen des deutschen Krankenhausinstitutes (2013) schaffen es nur $12,1 \%$ der Häuser auf ein ausgeglichenes Jahresergebnis. 42,2\% der Häuser beenden das Jahr 
mit einem Fehlbetrag und etwa dieselbe Menge an Krankenhäusern $(45,6 \%)$ erreichen einen Jahresüberschuss. Hier zeigen sich die Folgen einer Kostenreform besonders deutlich. Einige der Krankenhäuser haben es in der Zeit seit der Reform geschafft, den wirtschaftlichen Gedanken in das Krankenhaus zu integrieren, während andere Krankenhäuser es bisher nicht geschafft haben, den Krankenhausbetrieb wirtschaftlich auszurichten.

Diese Ausrichtung kann aus zwei Gründen recht schwierig sein. Zum einen trifft ein recht starres Vergütungssystem auf einen relativ wenig regulierten Markt von Krankenhäusern. Hier herrscht in einigen Bereichen hoher Wettbewerbsdruck, mit welchem einige Krankenhäuser nur schwer umgehen können. Zum anderen trifft der Gedanke der Wirtschaftlichkeit im Krankenhaus bei den Mitarbeitern nicht immer auf Verständnis und Wohlwollen. So sind einige Mitarbeiter wenig bereit dazu, sich dem Gedanken der Wirtschaftlichkeit in einem Krankenhaus zu stellen, da der Ursprungsgedanke des helfenden Berufes, nicht mit einer betriebswirtschaftlichen Sichtweise übereinzustimmen scheint (Staar et al. 2018).

Diese Erkenntnisse implizieren, dass es Unterschiede zwischen den Organisationen und auch den Mitarbeitern geben muss, da einige Krankenhäuser sehr erfolgreich am Markt agieren und andere Krankenhäuser nicht. Vor dem Hintergrund der Besonderheit der Organisation Krankenhaus, können nicht einfach Erkenntnisse der wirtschaftlichen Ausrichtung von anderen Organisationsformen übernommen werden.

\subsection{Studiendesign der Interviewbefragung}

Um herauszufinden, warum einige Krankenhäuser erfolgreich sind und andere Krankenhäuser nicht erfolgreich am Markt arbeiten, sollten diese Fragen mithilfe von halbstrukturierten Experteninterviews überprüft werden. Zum einen sollten mit der Erhebung intraindividuelle Ressourcen und Widerstände von wirtschaftlichem Arbeiten im Krankenhaus herausgearbeitet werden. Zudem sollte auch die Organisationsform betrachtet werden, welche unter Umständen wirtschaftliches Arbeiten in einigen Bereichen fördert oder durch bestimmte Strukturen behindert. Zuletzt sollte herausgefunden werden, in welchen Bereichen wirtschaftliches Arbeiten besonders stark vertreten ist und wie diese Verteilung zu erklären ist.

Um mit einer kleinen Erhebung möglichst viele Erkenntnisse zu generieren, wurden verschiedene Mitarbeiter aus verschiedenen Organisationen und Ebenen ausgewählt. In der finalen Stichprobe waren damit zwei Personen aus der Pflege, ein Assistenzarzt, zwei Chefärzte und ein Verwaltungsdirektor. Durch die verschiedenen Personen bei der Befragung konnten verschiedene Sichtweisen aus verschiedenen Organisationen zur wirtschaftlichen Ausrichtung ge-

neriert werden und in Verbindung mit der Theorie bewertet werden. Die Auswertung der Interviews erfolgte nach einer ausführlichen Transkription in Anlehnung an Mayring. Gefundene und gebildete Kategorien wurden dann mit theoretischen Erkenntnissen verglichen.

\subsection{Ergebnisse der Befragung}

Eine Zusammenfassung der theoretischen Gegenüberstellung in Kombination mit den Ergebnissen der Interviews in Bezug auf die Organisationsform findet sich in Tab. 1. Die Ergebnisse, die sich auf das Individuum beziehen, finden sich in Tab. 2.

Bei der Auswertung der Interviews konnte herausgefunden werden, dass auch die Mitarbeiter in einem Krankenhaus den Kostendruck und die Notwendigkeit einer Ausrichtung auf eine wirtschaftliche Arbeitsweise bemerken. Jedoch wurde auch bemängelt, dass die aktuelle Organisationsform einen Wandel der Ausrichtung nicht in allen Bereichen unterstützt. Dieser Wandel ist jedoch von höchster Relevanz. Es konnten einige Unterschiede zwischen Krankenhäusern und anderen Unternehmen herausgearbeitet werden. Hier erschienen besonders drei Punkte von besonderer Bedeutung. Zum einen ist dies die Ethikfrage, die in Bezug auf das unternehmerische Denken und Handeln gestellt werden muss. Wie weit darf und kann ein Mitarbeiter in einem Krankenhaus unternehmerisch denken und ab wann beeinflusst dieses Denken und Handeln den Patienten negativ. Die zweite Besonderheit bilden die Patienten. Hier

Tab. 1 Ergebnisse aus den Interviews und der Theorie - Organisation

Management Support

Unterstützung durch die Führungskraft

Entscheidungsfreiheit

Fordern und Fördern von unternehmerischem Denken

Vorbildfunktion durch die Führungskraft

Bereitstellung von Ressourcen

Zeitliche Freiräume

Vorbildfunktion durch die Füh-

rungskraft

Fachliche Begleitung

Weiterbildung

Fachlicher Austausch

Weiterbildung

Besuch von Kongressen

Personalentwicklung

\section{Kultur}

Offene Fehlerkultur

Risikotoleranz

Einfache Prozessgestaltung

Gegenseitige Unterstützung

Vertrauen und Wertschätzung

Sicherheit am Arbeitsplatz

Flexibilität

Bottom-Up Umsetzung zulassen

\author{
Kommunikation \\ Transparente Kommunikation \\ von erwarteten Verhaltensweisen \\ Prozesse im Krankenhaus erklä- \\ ren \\ Transparenz bei Zahlen \\ Einfache Kommunikationsstruk- \\ turen \\ Awareness schaffen \\ Pflege der Leitlinien \\ Leitlinien für unternehmerisches \\ Denken müssen von allen ge- \\ kannt und akzeptiert sein
}


Tab. 2 Ergebnisse aus den Interviews und der Theorie Individuum

Abb. 1 Eckpfeiler einer Corporate-Entrepreneurship-Strategie in Krankenhäusern. (Staar et al. 2018)

\begin{tabular}{|c|c|c|}
\hline Persönlichkeitsmerkmale & Kognitive Aspekte & Motivationale Aspekte \\
\hline $\begin{array}{l}\text { Risikoneigung } \\
\text { Ambiguitätstoleranz }\end{array}$ & $\begin{array}{l}\text { Mentale Fähigkeit } \\
\text { Hohes Selbstvertrauen } \\
\text { Wissen + Kenntnisse }\end{array}$ & $\begin{array}{l}\text { Leidenschaft } \\
\text { Self Efficacy }\end{array}$ \\
\hline Soziale Aspekte & Organisationale Aspekte & Umweltaspekte \\
\hline $\begin{array}{l}\text { Überzeugungskraft } \\
\text { Sozialkapital } \\
\text { Durchsetzungskompetenz } \\
\text { Kommunikationskompetenz }\end{array}$ & $\begin{array}{l}\text { Erstellung von Businessplänen } \\
\text { BWL Kenntnisse }\end{array}$ & Schulische Ausbildung \\
\hline
\end{tabular}

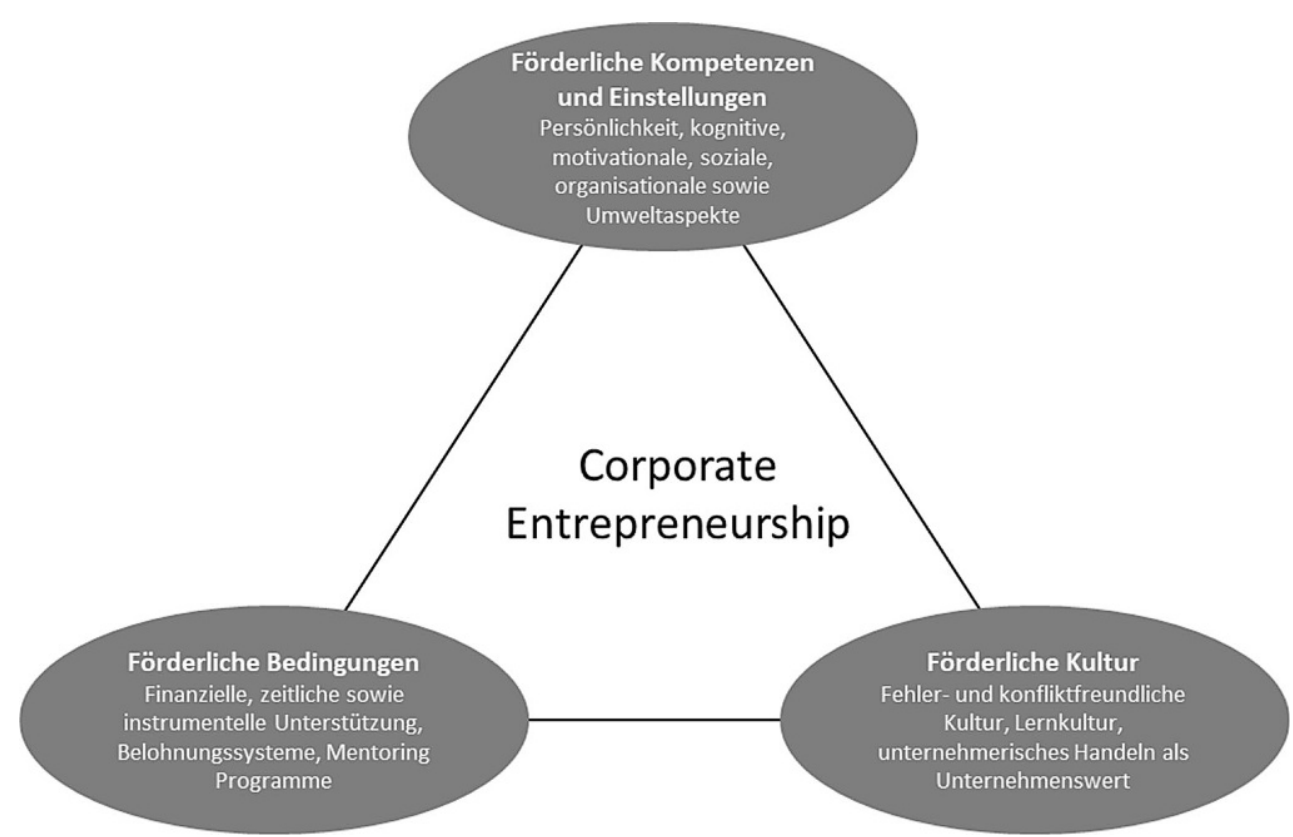

muss eine klare Unterscheidung von Patienten und Kunden vorgenommen werden, da Patienten sich in einer Not- bzw. Extremsituation befinden und auf die Hilfe des Personals angewiesen sind. Der dritte Punkt ist die Motivation der Mitarbeiter. Besonders in den Pflegeberufen scheinen hier der Altruismus und die Nächstenliebe ein bezeichnendes Motiv für die Arbeitsmotivation zu sein. Dieses Motiv erweist sich als äußerst positiv bei der Behandlung und Pflege der Patienten, könnte aber zugleich auch ein Hindernis bei unternehmerischem Denken und Handeln bedeuten. Natürlich handeln Mitarbeiter in der Pflege nicht allein aufgrund dieses Motivs. Es kommen weitere Anreize und Motive hinzu. Allerdings stellten alle Interviewteilnehmer dieses Motiv als ein wichtiges Motiv von Pflegekräften heraus.

Möchte man die Vorteile von unternehmerischem Denken und Handeln in einem Krankenhaus nutzen, bietet es sich an, diesen Grundgedanken in die Leitkultur der Organisation zu integrieren. Dies ist mit Corporate Entrepreneurship möglich. Hierbei handelt es sich um eine Unternehmenskultur, die Innovationen der Mitarbeiter fördert und umsetzt. Dadurch kann sich die Organisation Krankenhaus an neue Situationen anpassen und einen Mehrwert in der
Behandlung von Patienten erarbeiten. Um dieses unternehmerische Denken und Handeln in ein Krankenhaus zu integrieren, sind drei Eckpfeiler entscheidend (Abb. 1).

Zunächst spielen förderliche Kompetenzen und Einstellungen bei den Mitarbeitern in einem Krankenhaus eine große Rolle. Persönlichkeitsmerkmale, kognitive, motivationale und soziale Aspekte nehmen hierbei Einfluss auf die Person. Zudem bringen förderliche Bedingungen, wie finanzielle, zeitliche und instrumentelle Unterstützung und eine förderliche Kultur Mitarbeiter dazu, unternehmerisch zu denken und zu handeln (Kuratko 2015).

\section{Interkulturelle Herausforderungen im Krankenhaus}

Vor dem Hintergrund einer immer internationalen Arbeitswelt hat auch der „Umgang mit verschiedenen Kulturen“ im Krankenhaus einen immer stärkeren Einfluss auf die Mitarbeiter und auf die Patienten. Sowohl Patienten als auch Mitarbeiter kommen immer häufiger aus anderen Kulturkreisen und bringen damit nicht nur andere kulturelle Hin- 
tergründe, sondern auch sprachliche Herausforderungen in den Alltag der Krankenhäuser mit ein (Staar und Kempny 2019).

\subsection{Interkulturelle Herausforderungen im Team}

Interkulturelle Teams können durch kulturelle Diversität im Team profitieren, es kann allerdings auch zu negativen Effekten in einem Team durch kulturelle Diversität kommen. Es können in Teams positive als auch negative Effekte auf die Arbeitsleistung im Team gefunden werden (Guillaume et al. 2013; Stahl et al. 2010; Tang und Wang 2017; van Dick et al. 2008, p. 1466). Auch ist es möglich, dass kulturell diverse Teams innovativer (Roberge und van Dick, 2010, S. 297) und kreativer (Lu et al. 2017, S. 1; Roberge und Dick 2010, S. 297; Stahl et al. 2010) arbeiten. Gleichzeitig können aber auch Effekte wie Zufriedenheit im Team oder der Gruppenzusammenhalt sowohl in positiver oder negativer Ausprägung in einem Team auftreten (vgl. Stahl et al. 2010). Es können somit in kulturell diversen Teams durchaus unterschiedliche Effekte gefunden werden. Diese unterschiedlichen Effekte lassen sich durch verschiedene Einflussfaktoren in kulturell diversen Teams bis zu einem gewissen Punkt erklären.

Zum einen ist die Organisation dafür verantwortlich, wie gut interkulturelle Teams funktionierten. Hier kann ein klarer Einfluss der Organisationskultur gefunden werden. Speziell der Umgang der Organisation und die Offenheit gegenüber interkultureller Diversität ist hier entscheidend, damit Teams höhere Leistung erbringen können und weniger häufig bei der Arbeit fehlen (Guillaume et al. 2013; Hofhuis et al. 2012, S. 969). Neben der Organisationskultur ist auch die Führungskraft und deren Führungsverhalten bedeutsam für interkulturelle Teams und kann die Teamleistung signifikant steigern (Lu et al. 2017, S. 2; Somech 2006, S. 150). Auf der Teamebene ist die Sprache im Team und die Kommunikationsmöglichkeit untereinander wichtig, um Konflikten vorzubeugen und die Arbeitsleistung zu steigern (Stahl et al. 2010; Tenzer et al. 2017, S. 816). Zuletzt sind auch die einzelnen Personen im Team wichtig und deren Umgang und Einstellung gegenüber interkultureller Diversität (Tang und Wang 2017). Neben den Herausforderungen von Interkulturalität im Team ist aber auch die Interkulturalität zwischen Patienten und dem Krankenhauspersonal ein wesentlicher Punkt im Spannungsfeld der Interkulturalität im Krankenhaus.

\subsection{Interkulturelle Herausforderungen zwischen Personal und Patienten}

Diese interkulturelle Diversität im Patienten-Mediziner Kontakt kann aufgrund von verschiedenen Situationen $\mathrm{zu}$ einem Spannungsfeld werden. Aufgrund von sprach- licher, werte- oder deutungsbezogener Divergenz kann es zwischen Klinikpersonal und den Patienten und deren Angehörigen zu Missverständnissen und Problemen bei der Behandlung kommen (Staar und Kempny 2019). Verständigungsprobleme aufgrund von Sprache oder einer unterschiedlicheren Beschreibung der Symptomatik von Krankheiten können Barrieren darstellen (Penka et al. 2012). Auch unterschiedliche Rollenverständnisse von Mann und Frau oder andere Ansichten zu Behandlungsmethoden und Medikamenten können zu Konflikten in der Behandlung führen (Allemann-Ghionda und Bukow 2011; Grützmann et al. 2012; Ilkilic 2008).

Diese Barrieren sind durchaus zu durchbrechen und können gezielt von Krankenhäusern angegangen werden. Zum einen können durch angebotene Mentorenprogramme und Trainingseinheiten die Mitarbeiter auf Situationen vorbereitet werden und ein interkulturelles Verständnis von medizinischen Behandlungen kann im Gesamtkontext des Krankenhauses entwickelt werden (Allemann-Ghionda und Hallal 2011). Auch Mitarbeiter aus anderen Kulturkreisen können geschult werden, sodass sowohl sprachliche als auch kulturelle Barrieren im Patientenkontakt möglichst selten auftreten. Zudem scheint eine wesentliche Komponente dieser interkulturellen Patienten-MitarbeiterBeziehung die Ressource Zeit und das Verständnis von anderen Kulturen zu sein (Lindert et al. 2008). An dieser Stelle können ohne Unterstützung durch die Organisation Spannungsfelder für das Krankenhauspersonal entstehen. Mitarbeiter in einem Krankenhaus treffen täglich auf eine hohe Arbeitsbelastung und einen chronischen Zeitmangel. Kommen dann weitere Hürden auf die Mitarbeiter zu, kann dies zu einer Überlastung bei der Arbeit führen. Hier treffen die Behandlung des Patienten und die beschriebenen Barrieren aufeinander und die Belastung des Personals steigt weiter an.

\section{Stress bei Medizinern}

Aufgrund von diversen Einflussfaktoren wie den Arbeitszeiten, Extremsituationen, Spannungsfeldern wie Wirtschaftlichkeit und/oder kulturelle Barrieren, die auf Mediziner im Krankenhaus, aber auch bei Medizinern in anderen organisationalen Kontexten wirken, nimmt die Belastung durch Stressoren am Arbeitsplatz immer weiter zu (Rasch et al. 2017).

Demnach leiden Ärzte immer stärker unter Stress und den Folgen von Stress. Bedingt durch hohe Arbeitszeiten von teilweise mehr als $80 \mathrm{~h}$ und Belastungen durch schwere Schicksale (z.B. in der Onkologie), schätzen sich immer mehr Ärzte selbst als BurnOut gefährdet ein (Bergner 2010). 
Die Goethe-Universität Frankfurt am Main stellte in einer Untersuchung durch Arbeitsmediziner fest, dass mehr als die Hälfte aller im Krankenhaus tätigen Ärzte unter gesundheitsschädlichem Stress leiden (Bauer und Groneberg 2013). In der bundesweiten Studie der Autoren Jan Bauer und David Groneberg gingen die Ergebnisse von über 2000 Fragebögen ein. Als Ergebnis zeigt sich eine Stressprävalenz bei Assistenzärzten von $63,8 \%$, bei Fachärzten von $54,3 \%$, bei Oberärzten $46,0 \%$ und bei Chefärzten $24,6 \%$. Deutlich erkennbar ist das abnehmende Stressempfinden mit zunehmender, hierarchischer Stellung. Es scheint somit einen Zusammenhang mit bestehenden Entscheidungsspielräumen und dem Stressempfinden zu geben (Bauer und Groneberg 2013). Größere Entscheidungsspielräume führen demnach zu einem geringeren Stressempfinden. Während Assistenzärzte bezüglich des Arbeitsablaufes und der Dienstpläne mehr fremdbestimmt sind, können besonders Chefärzte die eigene Arbeit stärker selbstbestimmt gestalten. Nicht nur die Entscheidungsspielräume sind zentrale Aspekte bei der Entstehung von Stress. Ähnlich wie bei vielen andern Berufsgruppen zeigt sich auch unter Ärzten, dass der Rückhalt durch soziale Kontakte einen hohen Stellenwert besitzt (Zwack 2015). Eine Studie unter 220 Ärzten des Forschungsinstituts ,esanum“ zeigte, dass sich Ärzte durch Konflikte mit Kollegen offensichtlich erheblich gestresst fühlen (Hamberger 2013). Konflikte am Arbeitsplatz sind laut dieser Studie der Hauptgrund für Stress und besitzen einen großen Einflussfaktor auf das Stressempfinden. Zudem spielen auch das Konkurrenzdenken und der Drang nach Perfektion in dem besonderen Arbeitsumfeld Krankenhaus eine Rolle in Bezug auf das Stresslevel bei Medizinern.

Wenn Kollegen Arbeitsbelastungen nicht mittragen und Kollegialität verloren geht, kann der Druck durch hohe Arbeitsbelastung zu einem entscheidenden Stressor werden. Umgekehrt führt die soziale Unterstützung durch Vorgesetzte und Kollegen zu einer Entlastung (Keller et al. 2010b). Als ein Grund für den Stress unter Ärzten kann die hohe Arbeitsintensität gesehen werden (Bergner 2010).

Als zusätzlichen Stressfaktor, besonders für die jungen Ärzte, sehen Bauer und Groneberg (2013) den höheren Anteil an Wochenenddiensten, Nachtdiensten und Überstunden. Das hohe Arbeitsaufkommen kann zu dem Gefühl führen, eigenen Ansprüchen nicht mehr gerecht werden zu können, weil viele Tätigkeiten gleichzeitig erledigt werden müssen.

Keller et al. (2010a) unterteilen die Stressoren, die die ärztliche Tätigkeit betreffen, in mehrere Subkategorien. Dazu zählen z.B. Verwaltungsaufgaben und Dokumentationspflicht, eine hohe Arbeitsintensität bei wenig Gelegenheit zu Pausen, zusätzliche Belastungen durch Vorträge, ein hoher Zeit- und Termindruck und auch Personalmangel. Nach Bergner (2010) wird nicht der direkte Arzt-Patienten-Kon- takt als Stress empfunden, sondern Faktoren wie Zeitmangel, Zeitdruck, Beschränkung der Berufsfreiheit durch Gesetze, Angst vor Gerichtsverfahren oder Anzeigen, mangelnde Kollegialität, mangelnde Sicherheit der Anstellung und finanzielle Unsicherheit.

Zwack (2015) zeigt in einer Untersuchung weitere Stressoren für Ärzte auf. Zu den subjektiven Hauptstressoren zählen administrative Aufgaben, Bürokratie und finanzielle Sicherheit.

Auch die kassenärztliche Bundesvereinigung macht auf die hohe zeitliche Belastung von Ärzten durch bürokratischen Aufwand aufmerksam. Ein niedergelassener Arzt verbringt durchschnittlich 26\% seiner Arbeitszeit mit Bürokratie, auch bei einem Klinikarzt sind es mehr als zwei Stunden täglich (Flintrop und Korzilius 2012). Diese Zeit fehlt dem Mediziner für den Arzt-Patienten-Kontakt, also für die eigentlich medizinische Tätigkeit, für die er ausgebildet wurde. Dies hat eine Unzufriedenheit der Ärzte zufolge, da sie sich mit weitgehend fachfremden Tätigkeiten beschäftigen müssen, aber auch Unzufriedenheit beim Patienten, dem der Eindruck vermittelt wird, dass der Arzt wenig Zeit hat. „Im Arztberuf klaffen Antrittsmotive und Arbeitsalltag häufig weit auseinander" (Zwack 2015, S. 18).

Der ständige Umgang mit Extremsituationen, wie sterbenden Patienten und das Leid dieser oder der Angehörigen, kann für Mediziner aufgrund der hohen emotionalen Herausforderungen einen weiteren Stressor darstellen. Auch Bergner sieht eine starke Belastung der Ärzte unter der ständigen Konfrontation mit dem Leid anderer (Bergner 2010). Des Weiteren betont er als Stressoren die fehlende Anerkennung, Zeitdruck, Finanzdruck und ,häufige Störungen oder Unterbrechungen der eigentlichen Tätigkeit“" (Bergner 2010, S. 79).

Als mittelstark belastend stuft er mangelnde Informationen z.B. über neue gesetzliche Regelungen ein und hält auch das fehlende kollegiale Miteinander und Spannungen im Verhältnis zu den Patienten für mögliche Stress auslösende Faktoren.

\subsection{Exkurs: Demand-Control-Modell}

Karasek (1979) beschrieb das sogenannte „AnforderungsKontroll-Modell“. Dieses Modell betrachtet Zusammenhänge zwischen hohen psychischen Arbeitsanforderungen und Stress.

$\mathrm{Zu}$ den psychischen Arbeitsanforderungen gehören der Arbeitsumfang (quantitativ), die Arbeitsqualität, die Arbeitsgestaltung (Zeitdruck, Unterbrechungen, etc.) aber auch soziale Komponenten, wie das Verhältnis zu Mitarbeitern und Vorgesetzen (Friedel und Orfeld 2002).

Nach Karasek (1979) wirken hohe Arbeitsanforderungen Stress auslösend, wenn wenig Spielraum bezüglich der eigenen Entscheidungen besteht. 
Die „Kontrolle“ bezieht sich auf unterschiedliche Faktoren. Zum einen betrifft sie die Option, eigene Fähigkeiten anwenden und ausschöpfen und sich damit weiterentwickeln zu können. Darüber hinaus ist aber auch die Art der Entscheidungsspielräume zum Beispiel in Bezug auf Innovationen, Kreativität oder Arbeitsgestaltung von Bedeutung (Friedel und Orfeld 2002).

Ist eine hohe Arbeitsanforderung mit hohen Entscheidungsspielräumen gekoppelt, wirkt sich diese eher nicht als Stress auslösend, sondern motivationsfördernd aus (Karasek 1979). Die erhöhte Anforderung wird als eine positive Herausforderung gesehen. Sie wird zum Motor weiteren Handelns. Nicht unberücksichtigt bleiben darf dabei allerdings, dass die Anforderungen das individuelle Begabungspotenzial nicht überschreiten sollten. Ansonsten wird die Herausforderung leicht zur Überforderung.

Vor dem Hintergrund des Demand-Control-Modells sollen im weiteren Verlauf der Arbeit die Handlungsempfehlungen entwickelt und evaluiert werden.

\subsection{Studiendesign und -durchführung}

Um das Stressempfinden der Mediziner zu erheben und in den Kontext der Spannungsfelder einordnen zu können, wurde sich für eine quantitative Erhebung unter Medizinern entschieden. Insgesamt konnten 63 niedergelassene Ärzte aus dem Kreis Duisburg für eine solche Befragung gewonnen werden. Die Durchführung des Fragebogens fand zum größten Teil bei einer Konferenz in Papierform statt, weitere Mediziner konnten in den einzelnen Praxen zur Teilnahme bewegt werden.

Bei der Erhebung sollte zum einen erhoben werden, welche Stress auslösenden Faktoren niedergelassene Ärzte beeinflussen. Zum anderen sollte überprüft werden, in welchem Ausmaß die Teilnehmer der vorliegenden Stichprobe Resilienz aufweisen und durch welche Einflüsse sie geprägt wurden. Übergeordnetes Ziel ist die Generierung von Handlungsempfehlungen zum Aufbau und Erhalt von Resilienz.

Der Fragebogen umfasste 11 Skalen und 76 Items. Im Zentrum der Aufmerksamkeit standen die Skalen zu den Themenbereichen: Soziale Unterstützung durch Kollegen (ISAK-K, Keller et al. 2010b), Positives Denken (SCI, Satow 2012), Aktive Stressbewältigung (SCI, Satow 2012), Alkohol- und Zigarettenkonsum (SCI, Satow 2012), Soziale Unterstützung (SCI, Satow 2012), Erholungsverhalten (Jurkat 2010), Wertschätzung, Administrative Tätigkeiten, Stresssymptome (SCI, Satow 2012) und Resilienz (Leppert et al. 2008).

Die Testung der Reliabilität mithilfe von Cronbachs-Alpha ergaben bei allen Skalen ausreichende oder zufriedenstellende Werte. Bei der Skala Positives Denken $\alpha=0,802$, bei der Skala Soziale Unterstützung $\alpha=0,871$, bei Alkoholund Zigarettenkonsum $\alpha=0,716$, bei der Skala zur aktiven
Stressbewältigung $\alpha=0,817$, bei der sozialen Unterstützung durch Kollegen $\alpha=0,644$, bei der Skala zur Wertschätzung $\alpha=0,642$, bei der Skala Administrative Tätgkeiten $\alpha=0,695$ und bei den Skalen Stresssymptome $\alpha=0,905$ und Resilienz $\alpha=0,947$. Damit konnten die Skalen für die weitere Auswertung genutzt werden.

\subsection{Exkurs: Resilienz}

Der Begriff Resilienz beschreibt in der Psychologie die Fähigkeit, mit Krisensituationen oder anhaltenden äußeren Belastungen umzugehen. Hierbei liegt der Fokus auf der Widerstandsfähigkeit gegenüber solchen Beeinträchtigungen und Belastungen. Eine höhere Resilienz führt demnach zu einem geringeren Stressempfinden (Scharenhorst 2008). Es handelt sich somit um das Phänomen der psychischen Widerstandsfähigkeit.

Die American Psychological Association (Comas-Diaz et al. 2018) nennt einige weitere Faktoren, die mit Resilienz assoziiert werden. So ist die Fähigkeit, realistische Pläne zu gestalten und diese auch zur Durchführung zu bringen, ein Faktor für Resilienz. Auch die positive Sicht auf sich und ein gesundes Selbstbewusstsein in eigene Fähigkeiten und Stärken gehören dazu. Zudem spielen die Kommunikationsund die Problemlösefähigkeit eine wesentliche Rolle. Alle diese Faktoren können von Personen erlernt und entwickelt werden (Comas-Diaz et al. 2018).

Bergner geht auf Aspekte ein, die zu einer Resilienzsteigerung führen. Er nennt vier wesentliche Aspekte im Bezug auf die Berufsgruppe der Ärzte. Resilienz kann demnach durch ein bleibendes Interesse am eigenen Beruf, ein starkes Selbstbewusstsein, die Kenntnis und Akzeptanz über eigene Grenzen und die sich selbst zugeschriebene Bedeutsamkeit der eigenen Rolle als Mediziner gestärkt werden (Bergner 2010).

\subsection{Studienergebnisse}

Bei dieser Studie wurde eine multiple Regression gerechnet, um die Effekte von Soziale Unterstützung durch Kollegen, Positives Denken, Aktive Stressbewältigung, Alkohol- und Zigarettenkonsum, Soziale Unterstützung, Erholungsverhalten, Wertschätzung, Administrativen Tätigkeiten, Stresssymptomen auf Resilienz zu erkennen. Hierbei stellte sich heraus, dass nicht alle Effekte geeignet waren, um Resilienz vorherzusagen.

Das erste Modell der multiplen Regression wurde gerechnet, um Resilienz mithilfe der Variablen Administrativen Tätigkeiten, die Wertschätzung und der Alkohol- und Zigarettenkonsum vorherzusagen. Es konnte eine signifikante Regressionsgleichung gefunden werden $(\mathrm{F}(3,59)=6,457, p<0,005)$, mit einem $\mathrm{R}^{2}$ von 0,247 . Das zweite Modell der multiplen Regression wurde ebenfalls 
Tab. 3 Maßnahmen gegen das Spannungsfeld Stress im medizinischen Bereich, eigene Darstellung

\begin{tabular}{|c|c|}
\hline Dimensionen & Maßnahmen \\
\hline $\begin{array}{l}\text { Soziale Unterstützung } \\
\text { (Keller et al. 2010a; Zwack 2015) }\end{array}$ & $\begin{array}{l}\text { Aktivität in Gemeinden oder Vereinen } \\
\text { Bewusst Zeit mit Familie und Freunden verbringen } \\
\text { Fixe Termine für Treffen mit Freunden } \\
\text { Wochenendausflüge } \\
\text { Urlaub mit Familie und/oder Freunden } \\
\text { Gespräche suchen }\end{array}$ \\
\hline $\begin{array}{l}\text { Aktive } \\
\text { Stressbewältigung } \\
\text { (Kaluza 2012) }\end{array}$ & $\begin{array}{l}\text { Gezielt Ursachen für Stress eliminieren } \\
\text { Zeitplan aufstellen } \\
\text { SMARTe Ziele formulieren } \\
\text { Zeitmanagement } \\
\text { Kompetenzbereiche der Angestellten effizient einsetzen } \\
\text { Aufgaben delegieren } \\
\text { Wochenplanung erstellen } \\
\text { Arbeitsabläufe strukturieren }\end{array}$ \\
\hline Alkohol- und Zigarettenkonsum & $\begin{array}{l}\text { Alternativen suchen } \\
\text { Im Bedarfsfall professionelle Hilfe in Anspruch nehmen }\end{array}$ \\
\hline Soziale Unterstützung & $\begin{array}{l}\text { Kollegiale Kontakte knüpfen } \\
\text { Aktivität in berufspolitischen Gremien } \\
\text { Besuche von Qualitätszirkeln } \\
\text { Gespräche und Austausch suchen } \\
\text { Fallbesprechungen } \\
\text { Zusammenarbeit mit Kollegen suchen }\end{array}$ \\
\hline $\begin{array}{l}\text { Administrative } \\
\text { Tätigkeiten } \\
\text { (Zwack 2015) }\end{array}$ & $\begin{array}{l}\text { Sinnhaftigkeit in Verwaltungsarbeiten erkennen } \\
\text { Delegieren } \\
\text { Diktierprogramme nutzen } \\
\text { Sorgfältige Auswahl der Software } \\
\text { Praxisübergreifende Personalkraft einstellen }\end{array}$ \\
\hline $\begin{array}{l}\text { Wertschätzung } \\
\text { (Wagner-Link 2010; Zwack 2015) }\end{array}$ & $\begin{array}{l}\text { Wertschätzung ist immer wechselseitig } \\
\text { Zeit für Patienten nehmen } \\
\text { Patientenbefragung } \\
\text { „Beschwerdekasten“ } \\
\text { Mitarbeiterbefragung } \\
\text { Kritische Selbstreflektion } \\
\text { Dankbarkeit für Verständnis der Familie äußern }\end{array}$ \\
\hline Positives Denken & $\begin{array}{l}\text { Probleme als Herausforderung sehen } \\
\text { Das Positive in Stress sehen } \\
\text { Aus Verhalten in vergangenen Situationen lernen } \\
\text { Selbstreflektion } \\
\text { Akzeptanz } \\
\text { „positive Tagesrückschau“ }\end{array}$ \\
\hline $\begin{array}{l}\text { Erholungsverhalten } \\
\text { (Wagner-Link 2010; Zwack 2015) }\end{array}$ & $\begin{array}{l}\text { Freie Zeit sinnvoll nutzen } \\
\text { Hobbys nachgehen } \\
\text { Ausreichend Schlaf } \\
\text { Regelmäßiger Urlaub } \\
\text { Ruhetage einlegen }\end{array}$ \\
\hline
\end{tabular}

gerechnet, um Resilienz mithilfe der vorherigen Variablen zu berechnen. Allerdings wurde hier die Variable soziale Unterstützung zusätzlich mit aufgenommen. Es konnte auch hier eine signifikante Regressionsgleichung $(\mathrm{F}(4,58)=5,903, p<0,000)$, mit einem $\mathrm{R}^{2}$ von 0,289 gefunden werden. Obwohl die Vorhersagekraft durch die Variable soziale Unterstützung gesteigert wird, muss das zweite Modell kritisch betrachtet werden, da es zwischen der Variable soziale Unterstützung und den anderen drei Variablen höhere Korrelationen gibt.

Resilienz konnte in dieser Studie durch 4 Variablen zu einem gewissen Teil beschrieben werden. Die Variablen so- ziale Unterstützung, Administrative Tätigkeiten, die Wertschätzung und der Alkohol- und Zigarettenkonsum haben daher einen Einfluss auf die Resilienz von Medizinern. Es sollen allerdings im weiteren auch Maßnahmen für die anderen Variablen erarbeitet werden, da diese theoriegeleitet dennoch aufgenommen werden sollen.

\subsection{Abgeleitete Maßnahmen}

Auch der Bereich ,Stress und Arbeitsbelastung“" stellt deutlich ein großes Spannungsfeld heraus, welches Ärzte aber auch das medizinische Personal in einem Krankenhaus be- 
lasten. Es lassen sich Maßnahmen zur Resilienzsteigerung in einzelnen Bereichen finden, die dann den Mitarbeitern in einem Krankenhaus helfen können, eine Widerstandsfähigkeit gegenüber belastenden Situationen aufzubauen (Tab. 3).

\section{Retention Management im Krankenhaus}

Es konnten drei große Spannungsfelder identifiziert werden, welche Mitarbeiter in einem Krankenhaus beeinflussen und sowohl auf die Motivation, die Arbeitszufriedenheit als auch auf die psychische und physische Gesundheit Einfluss haben können. Während es sich bei dem Spannungsfeld „unternehmerisches Denken und Handeln“ eher um einen Bereich handelt, der Unternehmensführung und Unternehmenserfolg in den Vordergrund stellt, handelt es sich bei dem Spannungsfeld ,interkulturelle Herausforderungen" um eine Herausforderung, die direkten Einfluss auf die Patienten-Mitarbeiter-Beziehung oder die Teamarbeit hat. Während man sich dem Spannungsfeld der Wirtschaftlichkeit bis zu einem gewissen Punkt entziehen kann, ist dies bei dem Spannungsfeld der interkulturellen Herausforderungen nicht möglich. Zusätzlich zu diesen beiden eher globalen Spannungsfeldern in der Organisation Krankenhaus kommt für ein weiteres Spannungsfeld „Stress“ hinzu, welches in diesem Artikel besonders auf die Ärzte eingeht. Hierbei ist jedoch zu berücksichtigen, dass auch die anderen Berufsgruppen deutlich unter Stress stehen (Rasch et al. 2017).

Bei dem Thema Stress handelt es sich zunächst um eine sehr individuelle und persönliche Herausforderung, der sich jeder Mitarbeiter in einem Krankenhaus stellen muss. Jedoch sollte hierbei immer berücksichtigt werden, dass das Stressempfinden der Mitarbeiter auch in der Verantwortung der Organisation liegt, da hier Ressourcen bereitgestellt werden können.

Vor dem Hintergrund dieser Spannungsfelder, dem Fachkräftemangel und der vorhersehbaren Verschärfung dieser Problematik, drängt sich die Frage nach einer Möglichkeit auf, nicht nur die Spannungsfelder bis auf ein Möglichstes zu reduzieren, sondern auch die Mitarbeiter trotz alledem nachhaltig an das Unternehmen zu binden und sie für die Arbeit zu motivieren. Dies soll im Folgenden unter dem Blick des Retention Managements beschrieben werden.

\subsection{Retention Management}

Unternehmen die Retention Management in die Organisation integrieren, versuchen die individuellen Bedürfnisse und Wünsche der Mitarbeiter wahrzunehmen und die Mitarbeiter zu fördern und zu bestätigen. Dadurch erhoffen sich die Unternehmen, dass Mitarbeiter sich langfristig an das
Unternehmen binden und dem Unternehmen erhalten bleiben (Ulatowski 2013). Vorteile des Retention Managements sind dann nicht nur für das Unternehmen, sondern auch für die Mitarbeiter spürbar.

Für die Mitarbeiter kann ein gelungenes Retention Management zu Arbeitszufriedenheit, Commitment und einer gefestigten sozialen Identität führen. Dadurch resultieren verschiedene Effekte, wie beispielsweise ein gesteigertes Zugehörigkeitsgefühl, mehr Sicherheit, Orientierung und soziale Unterstützung untereinander. Effekte für ein Unternehmen können sinkende Fluktuationsraten, eine Leistungssteigerung der Mitarbeiter, ein größerer Personalpool, geringe Fehlzeiten und die Bindung von Wissen an das Unternehmen sein (Klaiber 2016).

Retention Management umfasst Faktoren, die einen Einfluss auf diese Effekte haben können. Zunächst sind hier die bereits genannten Faktoren Arbeitszufriedenheit, Commitment und die soziale Identität wesentlich. Diese Faktoren beeinflussen sich zudem auch untereinander und können sich dadurch gegenseitig verstärken (Ulatowski 2013).

Zudem wirken auf die Mitarbeiter weitere Faktoren ein, die eine Bindung an das Unternehmen verstärken oder schwächen können. Hier haben organisationale Faktoren und die Arbeitsbedingungen einen Einfluss. Während diese Faktoren durch ein Krankenhaus bis zu einem gewissen Punkt auf die Mitarbeiter abgestimmt werden können, sind Faktoren aus dem Arbeitsmarkt und Lebensfaktoren der Mitarbeiter weitaus weniger zu beeinflussen (Kanning 2017).

Wie können die Erkenntnisse über die Spannungsfelder in einem Krankenhaus für das Retention Management genutzt werden? Das große Problem für Krankenhäuser und die Belegschaft ist, dass sich neue Herausforderungen in den Krankenhausalltag integriert haben, die primär nichts mit dem Bild eines Mediziners oder von medizinischem Personal zu tun haben. So kommen auf alle Krankenhausangestellten immer mehr Fragen aus einem betriebswirtschaftlichen Blickwinkel zu, die diese nicht verstehen können oder wollen. Fehlt das Verständnis für Maßnahmen, die sich aus der wirtschaftlichen Ausrichtung des Krankenhauses ergeben, führt dies zu Unmut und Ablehnung dieser Maßnahmen. Da diese Maßnahmen aber mehr und mehr an Bedeutung gewinnen müssen, um ein Krankenhaus vor dem finanziellen Aus zu bewahren, ändern sich die Arbeitsanforderungen für das Krankenhauspersonal. Dies kann bei den Mitarbeitern zu einer geringeren Identifikation mit dem Unternehmen führen, obwohl die Identifikation gerade bei der Mitarbeiterbindung eine große Rolle spielt. $\mathrm{Zu}$ diesem ersten Spannungsfeld kommen auch die anderen Spannungsfelder mit starker Arbeitsbelastung, wenig Zeit und geringen Handlungsfreiräumen hinzu. Für die Mitarbeiter können so auch organisationale Faktoren und auch die Arbeitsbedingungen aus dem Gleichgewicht geraten. Vor dem 
theoretischen Hintergrund des Retention Managements und der Gegenüberstellung mit den Spannungsfeldern, scheint dann auch die hohe Fluktuation in einem Krankenhaus nicht mehr verwunderlich.

Es gibt jedoch einige Möglichkeiten, wie Krankenhäuser sich vor dieser hohen Fluktuation schützen und dann das Thema Mitarbeiterbindung in den Alltag des Krankenhauses integrieren können. Zunächst fängt hier die Arbeit bei den eigenen Mitarbeitern an. Die Mitarbeiter müssen in der Lage sein, mit den Spannungsfeldern zu arbeiten und die Arbeit so anzupassen, dass keine negative Beeinflussung der Arbeit durch die Spannungsfelder geschieht.

Beim ersten Spannungsfeld gibt es verschiedene erprobte Methoden, die Mitarbeitern helfen können, mit der Wirtschaftlichkeit im Krankenhaus umzugehen und diese zu verstehen. Gleiches trifft auch für das zweite Spannungsfeld zu. Hierfür konnte ein „Action Plan“ mit Hilfe von Experteninterviews und einer theoretischen Fundierung entwickelt werden, der diese beiden Spannungsfelder bearbeitet (Tab. 4).

Neben der konkreten Bearbeitung von Spannungsfeldern sind auch allgemeine Methoden des Retention Managements für ein Krankenhaus geeignet. Hier sollen die beiden angesprochenen, von dem Krankenhaus beeinflussbaren Faktoren Arbeitsbedingungen und organisationale Faktoren, betrachtet werden. Bei der Betrachtung von allgemeinen Methoden zur Steigerung der Mitarbeiterbindung ist immer zu beachten, dass es sich um ein besonderes Arbeitsumfeld handelt, bei dem die Mitarbeiter im Kontakt mit dem Patienten immer eine besondere und einzigartige Art von Dienstleistung erbringen und so Methoden, wie leistungsbedingte Vergütung immer kritisch vor dem
Tab. 4 Action Plan für die Spannungsfelder ,Wirtschaftlichkeit“" und „Interkulturalität“, eigene Darstellung

\author{
Kommunikation \\ Transparente Kommunikation \\ (vgl. Kuratko 2015)
}

Aufbau von einfachen Kommunikationsstrukturen

Kultur

Handlungsspielräume geben (Hornsby et al. 2002; vgl. Kuratko 2015)

Identität mit dem Krankenhaus

Erwünschte Verhaltensweisen in Bezug auf die Spannungsfelder werden klar kommuniziert

Organisatorische Richtlinien werden in Bezug auf die Dringlichkeit und Relevanz erklärt

Erfolge und Hindernisses werden zu den Spannungsfeldern kommuniziert

Regelmäßige Austauschveranstaltungen

Mentoren und Ansprechpartner für Probleme

Lockere Gesprächsrunden und Fachvorträge einzelner Mitarbeiter verbinden

Unterstützung der Kommunikation durch die Führungsetagen

Innovative Ideen müssen vom Management wahrgenommen und wertgeschätzt werden

Fehlerkultur entwickeln (hierbei ist darauf zu achten, dass das Patientenwohl immer an erster Stelle steht und nicht in einer Fehlerkultur beeinträchtigt werden darf)

Arbeitsplatzsicherheit

Arbeitgeber als Vertrauensperson (Ansprechpartner bei privaten Problemen)

Wertschätzende und vertrauensbasierte Führung

Individuelle Flexibilität bei Mitarbeiterbedürfnissen

Ethik (vgl. Deutscher Ethikrat 2006)

1. Die Behandlungsmethoden, die für einen Patienten ausgewählt werden, sollten nie durch Faktoren wie Umsatz oder Gewinn beeinflusst worden sein.

2. Eine medizinisch korrekte Behandlung steht immer vor unternehmerischem Verhalten durch die Mitarbeiter.

3. Unternehmerisches Denken darf nur dann angewendet werden, wenn dabei neutrale oder positive Effekte für den Patienten entstehen. Niemals dürfen negative Effekte in Kauf genommen werden.

4. Unternehmerisches Denken sollte nicht extra und individuell vergütet werden, sondern immer in einem gesamtunternehmerischen Rahmen belohnt werden. So schützt man Patienten vor möglichen voreiligen Umsetzungen, bedingt durch unternehmerisches Denken und Handeln

Weiterbildung

Personalentwicklung

(vgl. Solomon et al. 2013)

Plattformen bereitstellen
Mitarbeiter sollten auch in fachfremden Bereichen wie BWL geschult werden, um Unternehmensprozesse besser zu verstehen Soft-Skills wie Kommunikation, Durchsetzungsstärke, Stresstrainings und interkulturelles Wissen können Mitarbeitern helfen, den Spannungsfeldern zu begegnen

Austausch über Meetings, Konferenzen, Coaches, Messen und Kongresse 
Tab.5 Allgemeine Maßnahmen des Retention Managements (in Anlehnung an Kanning 2017)

\begin{tabular}{|c|c|c|}
\hline \multicolumn{3}{|c|}{ Allgemeine Maßnahmen für ein Retention Management in Krankenhäusern } \\
\hline \multirow[t]{4}{*}{$\begin{array}{l}\text { Arbeitsbedingungen } \\
\text { und -inhalte }\end{array}$} & $\begin{array}{l}\text { Passung der Arbeitsinhalte und } \\
\text { der Qualifikationen/Fähigkeiten/ } \\
\text { Interessen }\end{array}$ & $\begin{array}{l}\text { Schulungen zu verschiedenen krankenhausnahen und -fernen Themen (BWL, } \\
\text { VWL, Unternehmensführung) } \\
\text { Einsatzplanung und Mentorenprogramme } \\
\text { Karriereberatung und -planung }\end{array}$ \\
\hline & $\begin{array}{l}\text { Steigerung der Bedeutsamkeit der } \\
\text { Arbeitsaufgabe }\end{array}$ & $\begin{array}{l}\text { Bedeutsamkeit der Arbeitsaufgabe als medizinisches Personal ist meist klar } \\
\text { Bedeutsamkeit von Verwaltung und Dokumentationsaufgaben herausstellen } \\
\text { Information der Mitarbeiter über Unternehmensausrichtung }\end{array}$ \\
\hline & Verstärkter Einsatz von Feedback & $\begin{array}{l}\text { Rückmeldung über Behandlungsmethoden } \\
\text { Rückmeldung zu Abrechnungen, Dokumentation } \\
\text { Feedback über externe Konferenzen und Seminare } \\
\text { Mentorenprogramme }\end{array}$ \\
\hline & Steigerung der Autonomie & $\begin{array}{l}\text { Projektgruppen } \\
\text { Einsatzplanung } \\
\text { Unternehmerisches Denken und Handeln fördern }\end{array}$ \\
\hline \multirow[t]{3}{*}{$\begin{array}{l}\text { Organisationale } \\
\text { Faktoren }\end{array}$} & Führung & $\begin{array}{l}\text { Setzen von präzisen und anspruchsvollen Zielen } \\
\text { Einfordern von Leistung in einem fairen Rahmen } \\
\text { Partizipation }\end{array}$ \\
\hline & $\begin{array}{l}\text { Umgang mit Unzufriedenheit und } \\
\text { Konflikten }\end{array}$ & $\begin{array}{l}\text { Implementierung eines professionellen Beschwerdemanagements } \\
\text { Implementierung eines professionellen Konfliktmanagements } \\
\text { Regelmäßige Mitarbeiterbefragungen } \\
\text { Implementierung von Unternehmensleitlinien }\end{array}$ \\
\hline & Information & $\begin{array}{l}\text { Ständige und weitreichende transparente Information über Unternehmensziele, } \\
\text {-herausforderungen und -erfolge } \\
\text { Umgesetzte Ideen von Mitarbeitern veröffentlichen und honorieren }\end{array}$ \\
\hline
\end{tabular}

Hintergrund des Patientenwohls hinterfragt werden müssen (Tab. 5).

\section{Schlussbetrachtung}

Das Umfeld Krankenhaus ist ein besonderes System, in dem Mitarbeiter enorm hohen Arbeitsanforderungen und -belastungen gegenüberstehen. Dennoch ist die Leistungsbereitschaft vieler Mitarbeiter in diesem System bei ungünstigen Arbeitszeiten und einer mittelmäßigen Bezahlung hoch. Diese Leistungsbereitschaft lässt sich durch persönliche Motive und die Bedeutsamkeit des Gesamtauftrages eines Krankenhauses, der Behandlung von Patienten, in Teilen erklären. Dennoch müssen Krankenhäuser auf die Mitarbeiter achten und ihnen Unterstützung anbieten, mit neuen und bestehenden Herausforderungen im Krankenhaus umzugehen. Hier kann das Retention Management eine Methode darstellen, mit der die Krankenhausleitung das Personal an das eigene Unternehmen binden und Spannungsfelder im Krankenhausalltag verringern kann. Viele Methoden des Retention Managements sind bereits in Unternehmen erprobt und können auch im Krankenhaus angewandt werden, dennoch müssen viele Methoden zunächst vor einem medizinethischen Hintergrund geprüft werden, bevor diese in Krankenhäusern Anwendung finden können.

Funding Open Access funding provided by Projekt DEAL.
Open Access Dieser Artikel wird unter der Creative Commons Namensnennung 4.0 International Lizenz veröffentlicht, welche die Nutzung, Vervielfältigung, Bearbeitung, Verbreitung und Wiedergabe in jeglichem Medium und Format erlaubt, sofern Sie den/die ursprünglichen Autor(en) und die Quelle ordnungsgemäß nennen, einen Link zur Creative Commons Lizenz beifügen und angeben, ob Änderungen vorgenommen wurden.

Die in diesem Artikel enthaltenen Bilder und sonstiges Drittmaterial unterliegen ebenfalls der genannten Creative Commons Lizenz, sofern sich aus der Abbildungslegende nichts anderes ergibt. Sofern das betreffende Material nicht unter der genannten Creative Commons Lizenz steht und die betreffende Handlung nicht nach gesetzlichen Vorschriften erlaubt ist, ist für die oben aufgeführten Weiterverwendungen des Materials die Einwilligung des jeweiligen Rechteinhabers einzuholen.

Weitere Details zur Lizenz entnehmen Sie bitte der Lizenzinformation auf http://creativecommons.org/licenses/by/4.0/deed.de.

\section{Literatur}

Albrecht, D. M., \& Töpfer, A. (2017). Handbuch Changemanagement im Krankenhaus. Berlin, Heidelberg: Springer.

Allemann-Ghionda, C., \& Bukow, W.-D. (Hrsg.). (2011). Orte der Diversität. Wiesbaden: VS.

Allemann-Ghionda, C., \& Hallal, H. (2011). Interkulturalität, Gesundheit und Medizin. In C. Allemann-Ghionda \& W.-D. Bukow (Hrsg.), Orte der Diversität (S. 173-190). Wiesbaden: VS. https:// doi.org/10.1007/978-3-531-92087-0_11.

Bauer, J., \& Groneberg, D. A. (2013). Ärztlicher Disstress - eine Untersuchung baden-württembergischer Ärztinnen und Ärzte in Krankenhäusern [Distress among physicians in hospitals-an investigation in Baden-Württemberg, Germany]. Deutsche Medizinische Wochenschrift, 138(47), 2401-2406. https://doi.org/10.1055/ s-0033-1359859. 
Bayer, T. (2018). Ärztliche Dokumentationspflicht und Einsichtsrecht in Patientenakten: Eine Untersuchung zu den $\$$ 630f und $630 \mathrm{~g}$ $B G B$ mit Bezügen zum nationalen sowie europäischen Datenschutzrecht. MedR Schriftenreihe Medizinrecht. Berlin: Springer.

Behar, B.I., Guth, C., \& Salfeld, R. (2016). Modernes Krankenhausmanagement: Konzepte und Lösungen. Bd. 3. https://doi.org/10. 1007/978-3-642-36132-6.

Bergner, T.M.H. (2010). Burnout bei Ärzten: Arztsein zwischen Lebensaufgabe und Lebens-Aufgabe (2. Aufl.). Stuttgart: Schattauer. http://www.content-select.com/index.php?id=bib_view\& ean $=9783794582037$

Blum, K., Löffert, S., Offermanns, M., \& Steffen, P. (2013). Krankenhausbarometer: Umfrage 2013

Comas-Diaz, L., Luthar, S. S., Maddi, S. R., O’Neill, H. K., Saakvitne, K. W., \& Tedeschi, R. G. (2018). The road to resilience

Deutscher Ethikrat (2006). Patientenwohl als ethischer Maßstab für das Krankenhaus

Van Dick, R., van Knippenberg, D., Hägele, S., Guillaume, Y. R.F., \& Brodbeck, F. C. (2008). Group diversity and group identification: the moderating role of diversity beliefs. Human Relations, 61(10), 1463-1492. https://doi.org/10.1177/0018726708095711.

Flintrop, J., \& Korzilius, H. (2012). Bürokratie in Praxen und Krankenhäusern: Vom Versuch, den Alltag in Ziffern zu pressen. https:// www.aerzteblatt.de/archiv/124403/Buerokratie-in-Praxen-undKrankenhaeusern-Vom-Versuch-den-Alltag-in-Ziffern-zupressen. Zugegriffen: 26. Juni 2020.

Friedel, H., \& Orfeld, B. (2002). Das Anforderungs-Kontroll-Modell: Psychische Belastungen am Arbeitsplatz sind einfach zu ermitteln

Grützmann, T., Rose, C., \& Peters, T. (2012). Interkulturelle Kompetenz in der medizinischen Praxis. Ethik in Der Medizin, 24(4), 323-334. https://doi.org/10.1007/s00481-012-0223-7.

Guillaume, Y.R.F., Dawson, J.F., Woods, S.A., Sacramento, C.A., \& West, M. A. (2013). Getting diversity at work to work: What we know and what we still don't know. Journal of Occupational and Organizational Psychology, 86(2), 123-141. https://doi.org/ 10.1111/joop.12009.

Hamberger, B. (2013). Stress mit Kollegen macht Ärzte krank. https:// www.gesundheitsstadt-berlin.de/stress-mit-kollegen-machtaerzte-krank-1670/. Zugegriffen: 26. Juni 2020.

Haupt, A., \& Bouncken, R. B. (2014). Produktivitätssteigerung durch Fachkräftesicherung - Herausforderungen im Krankenhausbereich. In R.B. Bouncken (Hrsg.), Springer-Gabler Research. Dienstleistungsmanagement im Krankenhaus (S. 267-287). Wiesbaden: Springer Gabler. https://doi.org/10.1007/978-3-65805134-1_12.

Hofhuis, J., van der Zee, K. I., \& Otten, S. (2012). Social identity patterns in culturally diverse organizations: the role of diversity climate1. Journal of Applied Social Psychology, 42(4), 964-989. https://doi.org/10.1111/j.1559-1816.2011.00848.x.

Hornsby, J.S., Kuratko, D.F., \& Zahra, S. A. (2002). Middle managers' perception of the internal environment for corporate entrepreneurship: Assessing a measurement scale. Journal of Business Venturing, 17(3), 253-273. https://doi.org/10.1016/ S0883-9026(00)00059-8.

Ilkilic, I. (2008). Kulturelle Aspekte bei ethischen Entscheidungen am Lebensende und interkulturelle Kompetenz [Cultural aspects of ethical decisions at the end of life and cultural competence]. Bundesgesundheitsblatt, Gesundheitsforschung, Gesundheitsschutz, 51(8), 857-864. https://doi.org/10.1007/s00103-008-0606-6.

Jurkat, H. B. (2010). Gesundheitsförderung, Lebensqualität und Stressbewältigung im Medizinstudium. Dissertation. Giessen: Uni Giessen.

Kaluza, G. (2012). Gelassen und sicher im Stress: Das Stresskompetenz-Buch - Stress erkennen, verstehen, bewältigen (4. Aufl.). Berlin Heidelberg: Springer.

Kanning, U.P. (2017). Personalmarketing, Employer Branding und Mitarbeiterbindung: Forschungsbefunde und Praxistipps aus der
Personalpsychologie. Berlin, Heidelberg: Springer. https://doi. org/10.1007/978-3-662-50375-1.

Karasek, R. A. (1979). Job demands, job decision latitude, and mental strain: implications for job redesign. Administrative Science Quarterly, 24(2), 285. https://doi.org/10.2307/2392498.

Keller, M., Aulike, B., Böhmert, M., \& Nienhaus, A. (2010a). Explorative Studie zur Erfassung arbeitsbedingter Stressoren und Ressourcen von Klinikärztinnen und -ärzten. Journal Psychologie Des Alltagshandelns, 5(1), 39-50.

Keller, M., Bamberg, E., Böhmert, M., \& Nienhaus, A. (2010b). Entwicklung eines Instruments zur stressbezogenen Arbeitsanalyse für Klinikärztinnen und -ärzte (ISAK). Zeitschrift für Arbeitswissenschaft, 64(4), 337-353.

Klaiber, S. (2016). Organisationales commitment. Dissertation. Research

Kuratko, D.F. (2015). Corporate entrepreneurship: accelerating creativity and innovation in organizations. In C. Shalley, M. A. Hitt \& J. Zhou (Hrsg.), The oxford handbook of creativity, innovation, and entrepreneurship. New York: Oxford University Press. https://doi.org/10.1093/oxfordhb/9780199927678.013.0027.

Leiner, F. (2012). Medizinische Dokumentation: Grundlagen einer qualitätsgesicherten integrierten Krankenversorgung (6. Aufl.). Stuttgart: Schattauer. Lehrbuch und Leitfaden ; mit 24 Tabellen

Leppert, K., Koch, B., Brähler, E., \& Strauß, B. (2008). Die Resilienzskala (RS): Überprüfung der Langform RS-25 und einer Kurzform RS-13. Klinische Diagnostik und Evaluation., 1, 226-243.

Lindert, J., Priebe, S., Penka, S., Napo, F., Schouler-Ocak, M., \& Heinz, A. (2008). Versorgung psychisch kranker Patienten mit Migrationshintergrund [Mental health care for migrants]. Psychotherapie, Psychosomatik, Medizinische Psychologie, 58(3-4), 123-129. https://doi.org/10.1055/s-2008-1067360.

Lu, L., Li, F., Leung, K., Savani, K., \& Morris, M. W. (2017). When can culturally diverse teams be more creative? The role of leaders' benevolent paternalism. Journal of Organizational Behavior. https:// doi.org/10.1002/job.2238.

Penka, S., Schouler-Ocak, M., Heinz, A., \& Kluge, U. (2012). Interkulturelle Aspekte der Interaktion und Kommunikation im psychiatrisch/psychotherapeutischen Behandlungssetting. Mögliche Barrieren und Handlungsempfehlungen [Cross-cultural aspects of interaction and communication in mental health care. Barriers and recommendations for action]. Bundesgesundheitsblatt, Gesundheitsforschung, Gesundheitsschutz, 55(9), 1168-1175. https://doi org/10.1007/s00103-012-1538-8.

Rasch, D., Dewitt, T., \& Eschenbeck, H. (2017). Stress im Krankenhaus. Prävention Und Gesundheitsförderung, 12(4), 285-293. https://doi.org/10.1007/s11553-017-0593-2.

Roberge, M.-É., \& van Dick, R. (2010). Recognizing the benefits of diversity: when and how does diversity increase group performance? Human Resource Management Review, 20(4), 295-308. https://doi.org/10.1016/j.hrmr.2009.09.002.

Satow, L. (2012). Stress- und Coping-Inventar (SCI): Testmanual und Normen. https://www.drsatow.de/tests/stress-und-copinginventar/. Zugegriffen: 26. Juni 2020.

Scharenhorst, Julia (2008). Resilienz - Neue Arbeitsbedingungen erfordern neue Fähigkeiten. In Vorstand des Berufsverbandes Deutscher Psychologinnen und Psychologen e. V. (BDP) (Hrsg.), Psychische Gesundheit am Arbeitsplatz, in Deutschland (S. 51-54). Berlin.

Schmidt, K., Meyer, J.E., Liebeneiner, J., Schmidt, C.E., \& Hüttenbrink, K.B. (2012). Fachkräftemangel in Deutschland: Umfrage zu Erwartungen von Chefärzten an junge Mitarbeiter [The shortage of qualified staff in Germany: a survey on head physicians' expectations of young doctors]. $H N O, 60(2), 102-108$. https://doi. org/10.1007/s00106-011-2406-9.

Solomon, G., Frese, M., Friedrich, C., \& Glaub, M. (2013). Can personal initiative training improve small business success?: A longitudinal South African evaluation study. The International Journal 
of Entrepreneurship and Innovation, 14(4), 255-268. https://doi. org/10.5367/ijei.2013.0129.

Somech, A. (2006). The effects of leadership style and team process on performance and innovation in functionally heterogeneous teams. Journal of Management, 32(1), 132-157. https://doi.org/10.1177/ 0149206305277799.

Staar, H., \& Kempny, C. (2019). Internationalisierung des Personals und der Patienten: Interkulturelle Herausforderungen und Chancen für das Krankenhaus. In M. Pfannstiel, P. Da-Cruz \& V. Schulte (Hrsg.), Internationalisierung im Gesundheitswesen. Wiesbaden: Springer Gabler.

Staar, H., Grothe, M., Gebhardt, B., \& Dehne, H. (2014). „Für wen und mit wem arbeiten wir?": Mitarbeiter- und Patientenorientierung im Krankenhaus. In W. Hellmann \& S. Aydin-Saltik (Hrsg.), Gesundheitswesen in der Praxis. Krankenhausmanagement für leitende Ärzte (2. Aufl. S. 407-432). Heidelberg: medhochzwei.

Staar, H., Kempny, C., \& Atzpodien, H. C. (2018). Entrepreneurship im Gesundheitswesen: Zur Relevanz unternehmerischer Potenziale im Krankenhaus. In M. A. Pfannstiel, P. Da-Cruz \& C. Rasche (Hrsg.), Entrepreneurship im Gesundheitswesen I: Unternehmensgründung - Geschäftsideen - Wertschöpfung (S. 95-124). Wiesbaden: Springer.

Stahl, G. K., Maznevski, M.L., Voigt, A., \& Jonsen, K. (2010). Unraveling the effects of cultural diversity in teams: A meta-analysis of research on multicultural work groups. JIBS : the Journal of the Academy of International Business, 41(4), 690-709.

Tang, N., \& Wang, Y. (2017). Cross-cultural teams. In E. Salas, R. Rico \& J. Passmore (Hrsg.), The Wiley-Blackwell handbook of the psychology of team working and collaborative processes $(\mathrm{S}$. 219-242). Chichester: Wiley-Blackwell.

Tenzer, H., Terjesen, S., \& Harzing, A.-W. (2017). Language in international business: a review and agenda for future research. $M a$ nagement International Review, 57(6), 815-854. https://doi.org/ 10.1007/s11575-017-0319-x.

Ulatowski, H. (2013). Zukunftsorientiertes Personalmanagement in der ambulanten (Alten-)Pflege. Wiesbaden: Springer.

Wagner-Link, A. (2010). Leben lernen (6. Aufl.). Verhaltenstraining zur Stressbewältigung: Arbeitsbuch für Therapeuten und Trainer. [inklusive CD mit Arbeitsblättern], Bd. 231. Stuttgart: KlettCotta. http://www.socialnet.de/rezensionen/isbn.php?isbn=9783-608-89095-2
Zwack, J. (2015). Wie Ärzte gesund bleiben - Resilienz, statt Burnout. Bd. 2. Stuttgart, New York: Thieme.

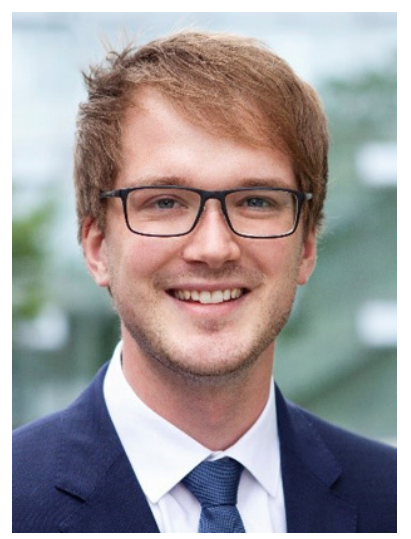

Christian Kempny M. Sc., studierte Business Psychology an der BiTS Business and Information Technology School GmbH in Iserlohn. Er arbeitet als wissenschaftlicher Mitarbeiter an der Hochschule HammLippstadt (HSHL) in dem landesgeförderten Forschungsprojekt $\mathrm{BeKu}$ Di - Benefits Kultureller Diversität. Zudem lehrt er im Fachbereich Organisationspsychologie an der Hochschule für Polizei und Verwaltung NRW. Aktuell strebt er eine Promotion im Bereich der Gesundheitswissenschaft an der Universität Witten/Herdecke an.

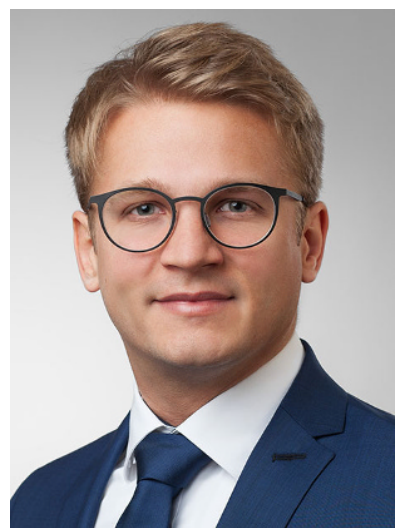

Christian Breimann M.Sc., studierte ebenfalls Business Psychology an der University of Applied Sciences Europe in Iserlohn. Er forschte im Zuge seiner Masterarbeit zum Thema: „Stressresilienz bei Ärzten im niedergelassenen Bereich“. Aktuell ist er als Junior Consultant bei der Unternehmensberatung Parodi \& Associates tätig. 